\title{
Design and simulation of a small capacity thermoacoustic refrigerator
}

\author{
Jakub Kajurek ${ }^{1}\left[\right.$ - Artur Rusowicz ${ }^{1}$. Andrzej Grzebielec ${ }^{1}$
}

(c) The Author(s) 2019 OPEN

\begin{abstract}
Thermoacoustic refrigerators use acoustic power to transport heat from a low-temperature reservoir to a high-temperature reservoir through a stack in a resonance tube. These devices nowadays can be considered as an alternative cooling technology as they use environmentally friendly working fluids and have few moving components. This paper presents the design procedure of a small thermoacoustic refrigerator with nominal cooling power of $10 \mathrm{~W}$. It explains the design choices with the reasons for using specific parameters. The design strategy applies simplified linear model of thermoacoustic in order to find optimum length and position of the stack. The model of the device was simulated by using a specialized tool DELTAEC. The simulation was performed under different temperatures of the cold heat exchanger varying from 250 to $295 \mathrm{~K}$, and under different drive ratios varying from 0.006 to 0.016 . The simulation results show that the cooling power of the refrigerator increases with the increase in the drive ratio and with the increase in the temperature of the cold heat exchanger. It was also found that the EER and the EERC coefficients of the device majorly depend on the temperature difference between the heat exchangers and only slightly depend on the drive ratio. The model achieved maximum cooling power of $55 \mathrm{~W}$ at the drive ratio of 0.016 and at the temperature difference between the heat exchangers of $5 \mathrm{~K}$, which corresponded to EER of 2.03 and EERC of 0.034 .
\end{abstract}

Keywords Thermoacoustics $\cdot$ Thermoacoustic refrigerator $\cdot$ Refrigerator $\cdot$ Cooling technology $\cdot$ Sound waves

\section{Introduction}

Concerns over environmental influence of hazardous refrigerants have impelled the researchers to searching and developing alternative cooling technologies as well as more environmentally friendly refrigerants. One of the most promising approaches in the class of new technologies is the thermoacoustic refrigeration [1, 2]. Thermoacoustic refrigerators are the machines which use sound to produce cooling power. They are mainly composed of an acoustic driver attached to an acoustic resonator which is filled with a working fluid and which encloses the heat exchangers and a stack. The thermal interaction between the acoustic waves that is sustained by the acoustic driver at a fundamental resonance frequency of the resonator with the stack material introduces an additional phase shift between the velocity and the temperature of the oscillating gas. This phase shift results in a longitudinal heat flux along the stack and enables transport heat from a cold to a hot reservoir, which is supplied to the cold end of the stack by the cold heat exchanger and is extracted from the hot end of the stack by the hot heat exchanger. A detailed explanation of the operating principles of the thermoacoustic devices is available in Swift review article [3].

Many prototypes of the thermoacoustic refrigerators have been developed since the success of the first cooler built by Hofler in 1986 [4]. The device driven by an acoustic driver at $500 \mathrm{~Hz}$ filled with the helium of 10 bar was capable of reaching $12 \%$ of a Carnot efficiency with an applied head load of $3 \mathrm{~W}$. A thermoacoustic refrigerator called Space ThermoAcoustic Refrigerator (STAR) was

Jakub Kajurek, jakub.kajurek@itc.pw.edu.pl | Institute of Heat Engineering, Faculty of Power and Aeronautical Engineering, Warsaw University of Technology, ul. Nowowiejska 21/25, 00-665 Warsaw, Poland.

SN Applied Sciences (2019) 1:579| https://doi.org/10.1007/s42452-019-0569-2

Received: 30 January 2019 / Accepted: 6 May 2019 / Published online: 17 May 2019 
designed and tested by Hofler and Atchley within the NASA's space shuttle program [5]. In January 1992, the STAR was flown on the Space Shuttle Discovery (STS-42). The cooling capacity of this unit with working frequency of $400 \mathrm{~Hz}$ was $3 \mathrm{~W}$ at a temperature drop of $50^{\circ} \mathrm{C}$, which corresponded to the EERC of $16 \%$. A thermoacoustic cooler constructed at Naval Postgraduate School in Monterey and supported by US Navy was installed on the ship USS Deyo (DD-989), where cooled ship's electronic radar [6]. The device named Shipboard Electronics Thermoacoustic Chiller (SETAC) was operating with helium-argon mixture at 21 bar and provided nearly $420 \mathrm{~W}$ of cooling power. The overall energy efficiency ratio in relation to Carnot coefficient was only $8.5 \%$. In 1996, the Office of Naval Research founded next project named TRITON, which aimed to develop a thermoacoustic chiller with cooling capacity of $10 \mathrm{~kW}$ [7]. The device constructed at Pennsylvania State University was completed in 2005 and reached to 19\% of Carnot cycle energy efficiency ratio with helium-argon gas mixture at 30 bar [8]. In 2000, Adeff and Hofler built and tested a thermoacoustically driven thermoacoustic refrigerator powered by solar thermal energy [9]. The goal of the project was to construct a compact unit capable of producing five to ten watts of cooling power at $0{ }^{\circ} \mathrm{C}$. An acoustic wave produced in the engine part of the system with a peak acoustic pressure amplitude of $4.3 \%$ allowed to achieve $2.5 \mathrm{~W}$ of cooling power at a cold temperature of $5^{\circ} \mathrm{C}$ and a temperature span of $18^{\circ} \mathrm{C}$. A similar device powered also by solar radiation was built by Chen in his Ph.D. thesis [10]. The cooler equipped in a 2-dimensional solar tracking system produced $6.2 \mathrm{~W}$ of cooling power at $28.5^{\circ} \mathrm{C}$.

The efficiency of the thermoacoustic devices is still not yet competitive against commercial solutions. Thus, many efforts have been taken in order to improve the design and the performance of the thermoacoustic coolers. The studies focus on the theoretical analysis as well as on the experimental and concern the optimization of the geometric parameters (e.g. stack position, stack length, resonator length) or the operating parameters (e.g. frequency, mean pressure, temperature gradient) [11]. Wetzel and Herman [12] developed a design algorithm for the thermoacoustic refrigerators based on the simplified linear thermoacoustic model with the normalized equations of the enthalpy flux and the work flux. The algorithm, for example, allows to select an optimum stack position and length at given temperature difference between the heat exchangers to get the maximum performance of the stack. A similar approach was also proposed by Tijani et al. [13] or Babaei and Siddiqui [14]. New optimization procedures such as genetic algorithm [15], particle swarm optimization [16], teaching-learning-based optimization algorithm
[17] and fruit fly optimization algorithm [18] were recently also introduced to the research area of thermoacoustics.

Most of the current experimental research has been done on the stack, where the thermoacoustic effect occurs. Tijani et al. [19] optimized the stack spacing for the maximum COP, the maximum cooling power and the lowest temperature. They found that the distance between the plates of about $2.5 \delta_{k}$ was optimum for the cooling power, a distance of $4 \delta_{k}$ gave the lowest temperature and a distance of $3 \delta_{k}$ provided the highest performance. Similar dependence for the lowest temperature was also observed by Setiawan et al. [20]. Another experimental optimization of porosity was performed by Yahya et al. [21], Tasnim et al. [22], Hariharan et al. [23] or Alcock et al. [24]. The stack location in resonance tube was also extensively studied as well in [25-28]. As it was found, the optimum position depends on the stack length. However, most of the favourable positions in these works fell between $\lambda / 20$ and $\lambda / 8$ measured away from the pressure antinode. In [29], Tijani et al. showed, in turn, that the performance of the thermoacoustic refrigerator improves as the Prandtl number of the working fluid decreases. Rest of the experimental studies concern the optimization of the operating parameters. Wantha et al. in [30] proved that the optimal operating frequency of a standing-wave thermoacoustic refrigerator differs from the design based on the equation $f=a / 2 L$, where $L$ is the resonator length and $a$ is the speed of sound. The influence of the operating frequency on the temperature difference created across the stack was also investigated in [31, 32], whereas, for example, in [33] the thermoacoustic refrigerator with the different stack geometry was examined under different cooling loads.

This work presents the design procedure of a small thermoacoustic refrigerator with the nominal cooling power of $10 \mathrm{~W}$ and with the temperature difference between the heat exchangers of $30 \mathrm{~K}$. The procedure details are explained step by step with the justification for the choice of the design parameters. The simplified linear thermoacoustic model was applied to find the optimum length and position of the stack. The designed device was also checked and simulated in the DELTAEC program under various temperatures of the cold heat exchanger and drive ratios in terms of cooling power, EER and EERC coefficients.

\section{Design of the thermoacoustic refrigerator}

The performance of the simplest thermoacoustic refrigerator depends on the eighteen independent parameters, which can be categorized into three groups [12] (Table 1). First includes geometrical parameters such as stack spacing and plate thickness, stack position and length, and cross-sectional area of the stack. Second group refers to 
Table 1 Parameters describing the thermoacoustic core

\begin{tabular}{lll}
\hline Geometrical parameters & Material specific parameters & Design requirements \\
\hline$L_{\mathrm{s}}$-stack length & Working fluid & $\dot{Q}_{\mathrm{C}}-$ cooling power \\
$x_{\mathrm{c}}$-stack centre position & $K$-thermal conductivity & $\Delta T_{\mathrm{m}}-$ desired temperature span \\
$2 y_{0}$-stack spacing & $a$-speed of sound & $T_{\mathrm{m}}$-mean operating temperature \\
$2 l$ - plate thickness & $\mu$-dynamic viscosity & $p_{\mathrm{m}}$-mean pressure \\
$A$-cross-sectional area & $\gamma$-ratio of isobaric to isochoric specific heats & $p_{1}$ pressure amplitude \\
& $\beta$-thermal expansion coeff. & $f$ frequency \\
& Stack material & \\
& $\rho_{\mathrm{s}}$-mean density & \\
& $c_{\mathrm{s}}$-specific heat & \\
& $K_{\mathrm{s}}$-thermal conductivity &
\end{tabular}

the material related variables which are thermophysical properties of the working fluid and the stack such as thermal conductivity or density. In the third group are designrelated parameters like frequency, mean pressure and pressure amplitude, mean temperature and temperature difference along the stack and cooling capacity of the system. The number of these independent parameters can be reduced trough normalization. The equations for the enthalpy flux $\left(\dot{H}_{\mathrm{n}}\right)$ and the work flux $\left(\dot{W}_{\mathrm{n}}\right)$ consumed by the stack can be then written as [14]:

$\dot{H}_{\mathrm{n}}=-\frac{1}{8 \gamma} \delta_{\mathrm{kn}}(\mathrm{DR})^{2} \frac{\sin \left(2 x_{\mathrm{cn}}\right)}{(1+\operatorname{Pr}) \Lambda}\left[\Gamma \frac{1+\sqrt{P r}+\operatorname{Pr}}{1+\sqrt{P r}}-\left(1+\sqrt{P r}-\delta_{\mathrm{vn}}\right)\right]$

$\dot{W}_{\mathrm{n}}=\frac{1}{4 \gamma} \delta_{\mathrm{kn}}(\mathrm{DR})^{2} L_{\mathrm{sn}}\left[\mathrm{BR}(\gamma-1) \cos ^{2}\left(x_{\mathrm{cn}}\right)\left(\frac{\Gamma}{(1+\sqrt{\operatorname{Pr}}) \Lambda}-1\right)-\frac{\sqrt{\operatorname{Pr}} \sin ^{2}\left(x_{\mathrm{cn}}\right)}{\mathrm{BR} \Lambda}\right]$ where

$\Gamma=\frac{\nabla T_{\mathrm{m}}}{\nabla T_{\text {crit }}}=\frac{\Delta T_{\mathrm{mn}} \tan \left(x_{\mathrm{cn}}\right)}{\operatorname{BR}(\gamma-1) L_{\mathrm{sn}}}$

$\Lambda=1-\delta_{\mathrm{kn}} \sqrt{\operatorname{Pr}}+\frac{1}{2} \operatorname{Pr} \delta_{\mathrm{kn}}^{2}$

The normalized parameters from Eqs. (1)-(4) with an extra index $n$ are shown in Table 2. The enthalpy flux $\left(\dot{H}_{\mathrm{n}}\right)$ is the sum of the work and the heat flux, and is constant, while the heat flux rises and the work flux drops. Using these principles, from Eqs. (1) and (2), the normalized cooling power can be expressed as:

$\dot{Q}_{\mathrm{Cn}}=\dot{H}_{\mathrm{n}}+\dot{W}_{\mathrm{n}}$

The EER coefficient of the stack is thus given by:
$\mathrm{EER}_{\mathrm{s}}=\frac{\dot{Q}_{\mathrm{Cn}}}{\left|\dot{W}_{\mathrm{n}}\right|}$

\subsection{Working fluid}

The working fluid for a thermoacoustic refrigerator should be characterized by high ratios of specific heats and low Prandtl numbers [34]. Therefore, the noble gases are recommended. Because of lower price in comparison with the other noble gases, the helium is chosen. Moreover, it has the highest sound velocity and thermal conductivity of all inert gases, which results in the higher cooling power and in the wider stack spacing, which in turn easier its construction.

\subsection{Average pressure}

The power density in a thermoacoustic refrigerator is proportional to the average pressure [3]. Thus, it is favourable to choose average pressure as large as possible. However, on the other hand there are factors that limit high pressure like the mechanical strength of the resonator and the effect of the pressure on the thermal penetration depth. High pressure requires a strong pressure vessel and very precise sealing, which very often lead to more expensive materials. High pressure also results in a small thermal penetration depth as it is inversely proportional to the square root of the average pressure. If the plate spacing is designed around the mean pressure, the problem can be countered by using very narrow gaps. However, the narrow gaps make the stack difficult to build with good precision. Thus, the choice of the average pressure must balance its influence on the power density, the resonator and the stack design. The average pressure of 10 bar is 
Table 2 Normalized parameters

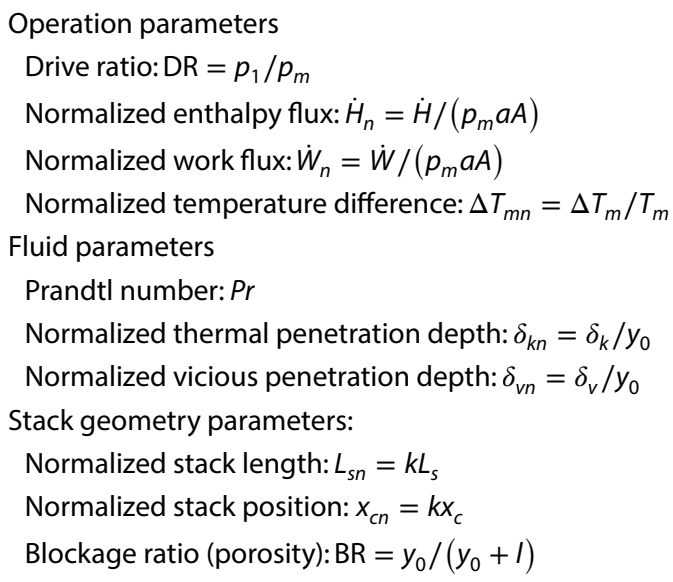

selected because combined with the frequency (Sect. 2.3) it allows to build a stack with reasonable size of the plate spacing and does not require any special material.

\subsection{Frequency}

The thermal penetration depth is inversely proportional to the square root of the frequency, so high resonance frequencies imply a narrow plate spacing. On the other hand, the power density in a thermoacoustic refrigerator is a linear function of the resonance frequency; therefore, high values are desired. The frequency also influences the length of the device as the resonator could be the quarter or the half-length of acoustic wave (Sect. 2.7); hence, low frequencies lead to long resonance tube. When the refrigerator is driven by a loudspeaker, the resonance frequency must also match the frequency response of the driver. Considering all these relationships, the frequency of $500 \mathrm{~Hz}$ is chosen as it allows to construct a compact device with the relatively wide plate spacing of the stack and enables to use a woofer as a sound source.

\subsection{Drive ratio}

The model of the thermoacoustic is based on the linear wave theory. In order to avoid the nonlinearities, the drive ratio should be smaller than 3\% so that the acoustic Reynolds number and the Mach number would be smaller than 500 and 0.1 , respectively [14]. The drive ratio should also be matched to the force of the acoustic driver. The drive ratios for the loudspeakers are on the order of a few per cent. To meet assumed cooling power without the need to drive the loudspeaker excessively hard, the drive ratio of 0.01 is selected.

\subsection{Stack}

The stack is the most important part of the thermoacoustic refrigerator, in which the heat-pumping process takes place. It should be made of a material characterized by a low thermal conductivity, which minimizes the losses caused by the ordinary heat conduction along the temperature gradient. The stack material should also have a heat capacity larger than the heat capacity of the working fluid, so that the temperature of the stack plates will stay steady [13]. The most commonly used effective material for the stack has been Mylar, as it has a low thermal conductivity and is produced in the wide range of thickness. For these reasons, this material is chosen for the stack.

There are many different stack geometries, such as parallel plates, circular pores, pin arrays and triangular pores. The main objective for the stack geometry is to increase the thermal boundary layer surrounding the solid wall where the thermoacoustic effect could occur [10]. Some of them are difficult to manufacture. Therefore, the stack geometry in the form of the parallel plates is selected. The optimal spacing between the plates for this type of the stack is between two and four thermal penetration depths [35]. The selection of helium as the working fluid, the average pressure of 10 bar and the frequency of $500 \mathrm{~Hz}$ determine the thermal penetration depth. Using the equation [2], we can obtain

$\delta_{k}=\sqrt{2 K /\left(\omega \rho_{\mathrm{m}} c_{\mathrm{p}}\right)}$

where $K$ is the thermal conductivity, $\omega$ is the angular frequency, $\rho_{\mathrm{m}}$ is the density and $c_{\mathrm{p}}$ is the isobaric heat capacity; the thermal penetration depth for designing conditions is equal to $0.107 \mathrm{~mm}$. Taking into account the above recommendations and simplicity of the fabrication, the plate spacing of $0.4 \mathrm{~mm}$ is selected.

The remaining parameters describing the stack, which are the stack centre position, stack length and stack cross-sectional area, will be chosen based on the optimization of the stack $E_{E R}$ (Eq. (6)). The calculations were done for eight stack centre positions, stack length varying from 30 to $160 \mathrm{~mm}$ and parameters presented in Table 3.

Table 3 Design parameters

\begin{tabular}{lll}
\hline Operation parameters & Fluid parameters & Stack parameters \\
\hline$p_{\mathrm{m}}=10 \mathrm{bar}$ & $\rho_{\mathrm{m}}=1.635 \mathrm{~kg} / \mathrm{m}^{3}$ & $K_{\mathrm{s}}=0.14 \mathrm{~W} / \mathrm{mK}$ \\
$T_{\mathrm{m}}=300 \mathrm{~K}$ & $C_{\mathrm{p}}=5.19 \mathrm{~kJ} / \mathrm{kgK}$ & $\rho_{\mathrm{s}}=1390 \mathrm{~kg} / \mathrm{m}^{3}$ \\
$\Delta T=30 \mathrm{~K}$ & $C_{\mathrm{v}}=3.12 \mathrm{~kJ} / \mathrm{kgK}$ & $2 l=0.1 \mathrm{~mm}$ \\
$f=500 \mathrm{~Hz}$ & $P r=0.662$ & $2 y_{0}=0.4 \mathrm{~mm}$ \\
$\mathrm{DR}=0.01$ & $K=0.154 \mathrm{~W} / \mathrm{mK}$ & \\
\hline
\end{tabular}




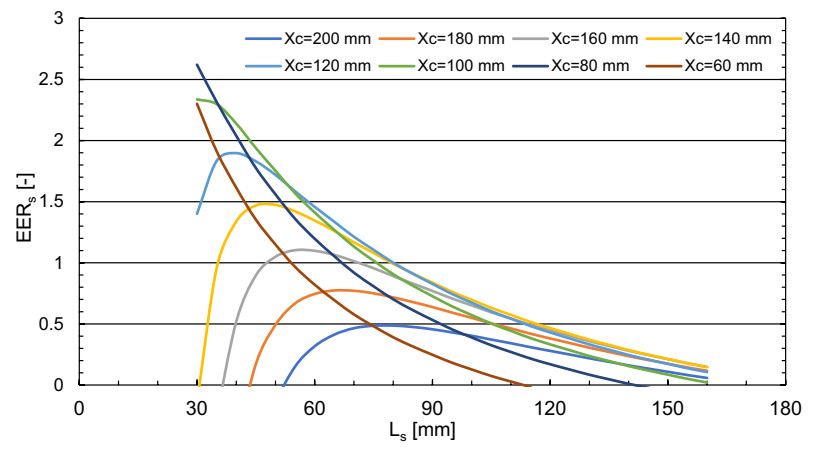

Fig. 1 EER of the stack versus the stack length for different stack centre positions measured from the sound source

Figure 1 shows that the $E \mathrm{R}_{\mathrm{s}}$ for each stack centre position increases with the stack length, reaches maximum and then starts to decrease. The performance peak shifts towards shorter plates together with the decrease in the stack centre position. Generally, the highest values of EER $\mathrm{E}_{s}$ are achieved by the shortest stacks located closer to the sound source. These positions correspond nearly to a pressure antinode and a velocity node where a small viscous loss occurs.

Taking into account described above relationships and for practical reasons, the length of $60 \mathrm{~mm}$ and the stack centre position at $120 \mathrm{~mm}$ away from the sound source are chosen. Among other configurations, these parameters show reasonable $E_{E R}$ of 1.45 . They will also facilitate the construction of the device by leaving enough space between the acoustic driver and the stack for heat exchanger and measurement equipment installation. The dimensionless cooling power for chosen parameter is equal to $1.05 \times 10^{-6}$. The required cooling power is assumed to be $10 \mathrm{~W}$. Using the equation for normalized cooling power (Eq. (5)) and the equations for normalized enthalpy and work flux presented in Table 2, the inner diameter of the large tube of the resonator $\left(D_{1}\right)$, which gives desired cooling power, should be of $108 \mathrm{~mm}$.

\subsection{Heat exchangers}

The proper design of the heat exchangers is a critical task in thermoacoustics, as the literature contains very little guidance [14]. Swift in [3] proposed the optimum length of a heat exchanger, which should be equal to peak-topeak gas displacement amplitude at the heat exchanger location. The displacement amplitude is equalled to [3]:

$x_{1}=\frac{p_{1}}{\omega \rho_{\mathrm{m}} a} \sin (k x)$

where $p_{1}$ is the pressure amplitude, $\omega$ is the angular frequency, $\rho_{\mathrm{m}}$ is the density, $a$ is the speed of sound, $k$ is the

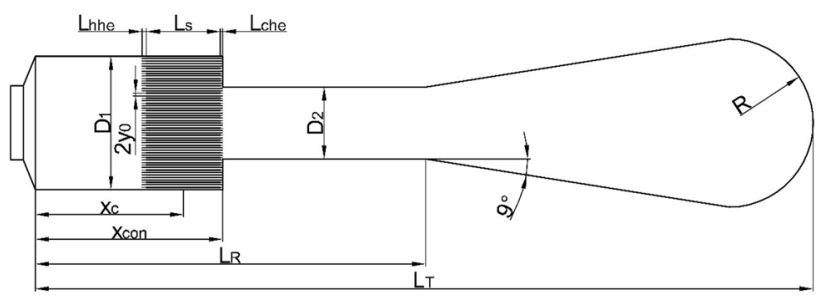

Fig. 2 Schematic illustration of the device

wave number and $x$ is the longitudinal coordinate. Using the above equation, designing conditions with the stack length and centre position the optimum length of the cold heat exchanger is about $1.8 \mathrm{~mm}$. The length of the hot heat exchanger could be calculated in the same way. However, the hot heat exchanger has to reject more heat than the heat supplied by the cold heat exchanger, as it also removes acoustic power used for the thermoacoustic effect. Assuming that the heat transfer coefficient and the temperature difference between the solid plate and the working fluid are the same, the length of the hot heat exchanger could be set as a twice length of the cold heat exchanger $(3.6 \mathrm{~mm})$. In order not to disturb the parcel displacement between the heat exchanger and the stack, the plate spacing of the both heat exchangers is identical as for the stack $\left(2 y_{0}=4 \mathrm{~mm}\right)$.

\subsection{Resonator}

The resonator length must match the resonance frequency of the system. In most cases, the resonance tubes are the quarter or the half-length of acoustic wave. The length of the resonator influences also the viscous and the thermal penetration losses. The total dissipated energy is proportional to the internal surface area of the resonator [3]. Hence, the quarter wave length resonator will dissipate half of energy dissipated in the half-length wave resonator. The shape of the resonator can be further optimized by minimizing its internal surface area. As it was shown by Hofler [4], this can be achieved by reducing the diameter of the resonance tube that is behind the stack and using a spherical bulb to simulate an open end. The total loss of energy has a minimum at about $D_{2} / D_{1}=0.54$ [13], where $D_{2}$ is the diameter of the small tube and $D_{1}$ is the diameter of the large tube containing the stack. Using this relationship, the inner diameter of the small tube should be of $58 \mathrm{~mm}$.

The total length of the resonator is equalled to the sum of the lengths of the small diameter tube and the large diameter tube (Fig. 2) and is determined by earlier chosen operation frequency of $500 \mathrm{~Hz}$. The length of each tube can be obtained considering the condition that at the junction of the small and the large diameter tubes the pressure and the 
volume flow rate have to be continuous. This implies also the same acoustic impedances at the connection of these tubes, which is reflected by the equation [13]:

$$
\cot \left(k x_{\text {con }}\right)=\left(\frac{D_{1}}{D_{2}}\right)^{2} \tan \left(k\left(L_{R}-x_{\text {con }}\right)\right)
$$

where $k$ is the wave number, $x_{\text {con }}$ is the longitudinal coordinate of the connection, $L_{R}$ is the total length of the resonator, $D_{1}$ is the diameter of the large tube and $D_{2}$ is the diameter of the small tube (Fig. 2). The total length of the resonator after substitution of all necessary parameters in Eq. (9) is equalled to $322.6 \mathrm{~mm}$. This value means that for the resonator with variable cross-sectional area its total length does not correspond to an integer number of the fundamental mode.

The buffer volume must have enough volume to simulate an open end. A spherical bulb firstly designed by Hofler [4] generates turbulence during the transition from the small diameter tube to the bulb. Tijani [36] proved that the minimal losses occur for the cone-shaped buffer volume with the half-angle of the cone of $9^{\circ}$. Therefore, a similar buffer tube with the volume of $2.5 \mathrm{~L}$ will be used. The volume determines the total length of the device $\left(L_{T}\right)$ to be $636 \mathrm{~mm}$ with the buffer radius (R) of $68 \mathrm{~mm}$ (Fig. 2).

\subsection{Acoustic driver}

The total acoustic power $\left(\dot{W}_{\mathrm{t}}\right)$ that must be provided by the acoustic driver is the sum of the acoustic power used by stack $\left(\dot{W}_{s}\right)$ to transfer heat and acoustic power dissipated in different parts of the device:

$\dot{W}_{\mathrm{t}}=\dot{W}_{\mathrm{s}}+\dot{W}_{\text {che }}+\dot{W}_{\text {hhe }}+\dot{W}_{\text {res }}$

The acoustic powers used by the stack, dissipated in the cold heat exchanger $\left(\dot{W}_{\text {che }}\right)$ and in the hot heat exchanger $\left(\dot{W}_{\text {hhe }}\right)$, are described by Eq. (2) and are equalled, respectively, to $\dot{W}_{\mathrm{s}}=6.86 \mathrm{~W}, \dot{W}_{\text {che }}=0.31 \mathrm{~W}, \dot{W}_{\text {hhe }}=0.48 \mathrm{~W}$. In the large diameter resonator, only the part of the tube does not contain any parts dissipating energy, and in the small diameter resonator, the energy is dissipated along all tube. In both cases, the amount of dissipated acoustic power can be estimated using the equation for normalized acoustic power dissipated in wavelength resonator with appropriate length's coefficient for given tube [14]:

$\Delta \dot{W}_{n}=-z\left[\left(\frac{\pi}{8 \gamma} \sqrt{\operatorname{Pr} \mathrm{DR}^{2}}\left(\frac{\delta_{k}}{R_{\mathrm{hr}}}\right)\right)-\left(\frac{\pi(\gamma-1)}{8 \gamma} \mathrm{DR}^{2}\left(\frac{\delta_{k}}{R_{\mathrm{hr}}}\right)\right)\right]$

where $z$ is the length coefficient, $\gamma$ is the ratio of isobaric to isochoric specific heats, $P r$ is the Prandtl number, DR is the drive ratio, $\delta_{k}$ is the thermal penetration depth and
$R_{\mathrm{hr}}$ is the hydraulic radius. The total acoustic dissipated in resonator is $\dot{W}_{\text {res }}=0.11 \mathrm{~W}$. Hence, the acoustic driver must deliver 7.76 W. The EER coefficient of the device described as the cooling power to the acoustic power delivered by the driver is 1.29. This coefficient does not include the efficiency of the driver; therefore, the final EER can be expected much smaller.

\section{Simulation}

The computer program Design Environment for Lowamplitude ThermoAcoustic Energy Conversion (DELTAEC) was used to simulate the designed device (Fig. 2). DELTAEC is the numerical tool developed by Los Alamos National Laboratory, which solves the one-dimensional wave equation in the gas based on the low-amplitude acoustic approximation in a geometry given by the user as a sequence of segments such as ducts, cones, stacks and heat exchangers [37]. The code uses a shooting method, by guessing and adjusting any unknown boundary conditions at the initial segment to achieve the desired boundary conditions elsewhere.

In the simulation, the volume flow amplitude, the mean temperature, the frequency and the heat removed from the hot heat exchanger were set as the guess parameters, and the real part of the inverse of the normalized specific impedance, the imaginary part of the inverse of the normalized specific impedance and the total energy flow determined at the final segment of the device were set as the target parameters as well as the temperature of the hot heat exchanger. The comparison between the results from design procedure and DELTAEC simulation is presented in Table 4. A good agreement can be observed. The small differences between these two ways are mainly due to assumptions that were made in the normalization of the thermoacoustic enthalpy flux and work flux.

Figures 3, 4 and 5 show the simulations results of the device for different temperatures of the cold heat exchanger $\left(T_{\text {che }}\right)$ and drive ratios (DR). The temperature of the hot heat exchanger $\left(T_{\text {hhe }}\right)$ and mean pressure $\left(p_{\mathrm{m}}\right)$ during the simulations was kept constant, respectively, at

Table 4 Comparison between results from design procedure and DELTAEC simulation

\begin{tabular}{ll}
\hline Design procedure & Simulation results \\
\hline$f=500 \mathrm{~Hz}$ & $f=505.93 \mathrm{~Hz}$ \\
$T_{\text {che }}=270 \mathrm{~K}$ & $T_{\text {che }}=268 \mathrm{~K}$ \\
$T_{\text {hhe }}=300 \mathrm{~K}$ & $T_{\text {hhe }}=300 \mathrm{~K}$ \\
$\dot{Q}_{\mathrm{c}}=10 \mathrm{~W}$ & $\dot{Q}_{\mathrm{c}}=10 \mathrm{~W}$ \\
$\dot{W}=7.76 \mathrm{~W}$ & $\dot{W}=7.83 \mathrm{~W}$ \\
EER $=1.29$ & EER $=1.28$ \\
\hline
\end{tabular}




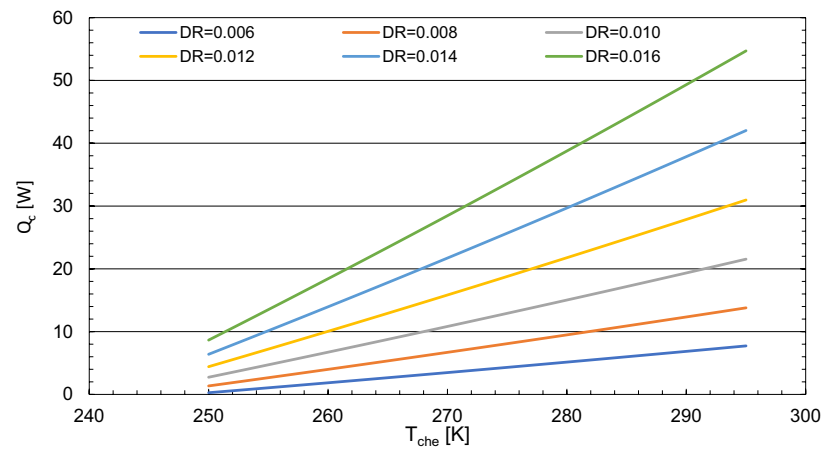

Fig. 3 Cooling power of the device versus temperature of the cold heat exchanger for different drive ratios

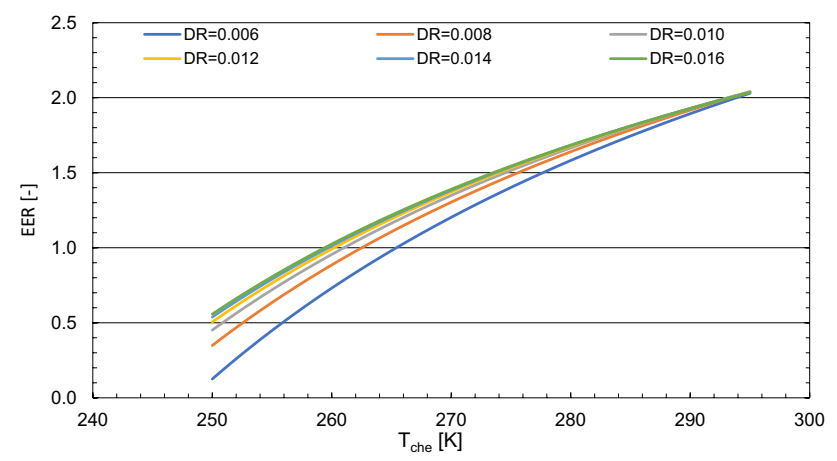

Fig. 4 EER of the device versus temperature of the cold heat exchanger for different drive ratios

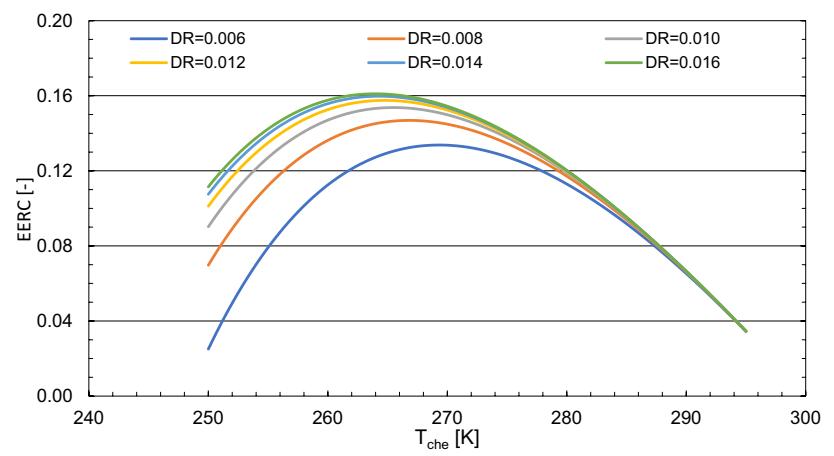

Fig. 5 EERC of the device versus temperature of the cold heat exchanger for different drive ratios

$300 \mathrm{~K}$ and $10 \mathrm{bar}$. The cooling power of the device increases with the increase in the drive ratio and with the increase in the cold heat exchanger temperature (Fig. 3). For hightemperature differences between the heat exchangers and for low drive ratios, the cooling power is nearly approaching zero. This is a consequence of the decrease in the critical temperature gradient (Eq. 3). The critical temperature gradient is a value for which the temperature change due to adiabatic compression and expansion matches the temperature change due to parcel motion of the working fluid along the stack plate [3]. The increase in the mean temperature gradient corresponds always to the increase in the temperature changes of the gas parcel caused by its displacement due to acoustic oscillation, what finally lead to the decrease in the heat flux. Below a certain ratio of the mean temperature gradient to the critical temperature gradient, a noxious effect starts to occur in which the acoustic energy is absorbed, and the heat is pumped from the hot to the cold heat exchanger (no cooling power). Further decrease in the cold heat exchanger temperature in simulation procedure would lead to such effect.

The effects of the temperatures of the cold heat exchanger and drive ratios on the EER coefficient are shown in Fig. 4. The EER increases with the increase in the temperature of the cold heat exchanger and with the increase in the drive ratio. However, the influence of the drive ratio, especially for small temperature span along the stack, is not so significant. Such behaviour of EER is expected because as Eqs. (1) and (2) show, both the enthalpy and the work flux are proportional to the square of the drive ratio. For lower temperatures of the cold heat exchanger, the effect of the drive ratio is more observable. This is due to low cooling power for small acoustic pressure amplitude (Fig. 3).

The EER coefficient relative to Carnot coefficient shows a parabolic dependence for all drive ratios and temperatures of the cold heat exchangers (Fig. 5). The maximum values of EERC exist because, while EER rises almost linearly with the temperature (Fig. 4), the EER ${ }_{C}$ of the Carnot cycle increases faster. Like the EER, the EERC increases with the increase in the drive ratio for high-temperature differences between the heat exchangers and is nearly similar for all drive ratios at small temperature differences. The peak of EERC shifts towards lower temperatures of the cold heat exchangers together with the increase in the drive ratio.

\section{Conclusion}

In this paper, the design procedure of a small capacity thermoacoustic refrigerator with the nominal cooling power of $10 \mathrm{~W}$ and with the temperature difference between the heat exchangers of $30 \mathrm{~K}$ is described. The design with the explanation of the choice of the design parameters is based on the dimensionless linear thermoacoustic model, which was used for the stack optimization. The algorithm was checked by the computer code DELTAEC, and a good agreement was achieved. The performance of the designed refrigerator under different temperatures of 
the cold heat exchanger and drive ratios was simulated in the program as well. The results show how sensitive on the change of the operational parameters the thermoacoustic refrigerator is. For example, an inappropriate temperature difference selection could lead to the change of the heat transport direction between the heat exchangers which will result in no cooling effect. It was also found that for analysed parameters the influence of the drive ratio on the EER and EERC coefficients especially for small temperature span, less than $15 \mathrm{~K}$, is almost negligible.

\section{Compliance with ethical standards}

Conflict of interest The authors declare that they have no conflict of interest.

Open Access This article is distributed under the terms of the Creative Commons Attribution 4.0 International License (http://creativeco mmons.org/licenses/by/4.0/), which permits unrestricted use, distribution, and reproduction in any medium, provided you give appropriate credit to the original author(s) and the source, provide a link to the Creative Commons license, and indicate if changes were made.

\section{References}

1. Herman C, Travnicek Z (2006) Cool sound: the future of refrigeration? Thermodynamic and heat transfer issues in thermoacoustic refrigeration. Heat Mass Transf 42:492-500. https://doi. org/10.1007/s00231-005-0046-x

2. Cyklis $P$, Janisz K (2015) An innovative ecological hybrid refrigeration cycle for high power refrigeration facility. Chem Proc Eng 36:321-330. https://doi.org/10.1515/cpe-2015-0022

3. Swift GW (1998) Thermoacoustic engines. J Acoust Soc Am 84:1145-1180. https://doi.org/10.1121/1.396617

4. Hofler TJ (1986) Thermoacoustic refrigerator design and performance. Dissertation, University of California

5. Garrett SL, Adeff JA, Hofler TJ (1991) A thermoacoustic refrigerator for space applications. J Acoust Soc Am 90:595-599. https:// doi.org/10.1121/1.401096

6. Ballister S, McKelvey D (1995) Shipboard electronics thermoacoustic cooler. Dissertation, Naval Postgraduate School

7. Johnson RA, Garrett SL, Keolian RM (2000) Thermoacoustic cooling for surface combatants. Nav Eng J 112:335-345. https://doi. org/10.1111/j.1559-3584.2000.tb03340.x

8. Girgin I, Türker M (2012) Thermoacoustic systems as an alternative to conventional coolers. J Nav Sci Eng 8:14-32

9. Adeff JA, Hofler TJ (2000) Design and construction of a solarpowdered, thermoacoustically driven, thermoacoustic refrigerator. J Acoust Soc Am 107:37-42. https://doi.org/10.1121/1.42899 3

10. Chen RL (2001) Design, construction, and measurement of a large solar powered thermoacoustic cooler. Dissertation, The Pennsylvania State University

11. Zolpakar NA, Mohd-Ghazali, Hassan El-Fawal M (2016) Performance analysis of the standing wave thermoacoustic refrigerator: a review. Renew Sustain Energy Rev 54:626-634. https://doi. org/10.1016/j.rser.2015.10.018
12. Wetzel M, Herman C (1997) Design optimization of thermoacoustic refrigerators. Int J Refrig 20:3-21. https://doi. org/10.1016/S0140-7007(96)00064-3

13. Tijani MEH, Zeegers JCH, De Waele ATAM (2002) Design of thermoacoustic refrigerators. Cryogenics 42:49-57. https://doi. org/10.1016/S0011-2275(01)00179-5

14. Babaei H, Siddiqui K (2008) Design and optimization of thermoacoustic devices. Energy Convers Manag 49:3585-3598. https:// doi.org/10.1016/j.enconman.2008.07.002

15. Zolpakar NA, Mohd-Ghazali N, Ahmad R (2016) Experimental investigations of the performance of a standing wave thermoacoustic refrigerator based on multi-objective genetic algorithm optimized parameters. Appl Therm Eng 100:296-303. https:// doi.org/10.1016/j.applthermaleng.2016.02.028

16. Chaitou H, Nika P (2012) Exergetic optimization of a thermoacoustic engine using the particle swarm optimization method. Energy Convers Manag 55:71-80. https://doi.org/10.1016/j. enconman.2011.10.024

17. Rao RV, More KC, Taler J, Ocłoń P (2017) Multi-objective optimization of thermo-acoustic devices using teaching-learning-based optimization algorithm. Sci Technol Built Environ 23:1244-1252. https://doi.org/10.1080/23744731.2017.12963 19

18. Rahman AA, Zhang X (2018) Single-objective optimization for stack unit of standing wave thermoacoustic refrigerator through fruit fly optimization algorithm. Int J Refrig 98:35-41. https://doi. org/10.1016/j.ijrefrig.2018.09.031

19. Tijani MEH, Zeegers JCH, De Waele ATAM (2002) The optimal stack spacing for thermoacoustic refrigeration. J Acoust Soc Am 112:128-133. https://doi.org/10.1121/1.1487842

20. Setiawan I, Bambang Setio Utomo A, Katsuta M, Nohtomi M (2013) Experimental study on the influence of the porosity of parallel plate stack on the temperature decrease of a thermoacoustic refrigerator. J Phys Conf Ser. https://doi. org/10.1088/1742-6596/423/1/012035

21. Yahya SG, Mao X, Jaworski J (2017) Experimental investigation of thermal performance of random stack materials for use in standing wave thermoacoustic refrigerators. Int J Refrig 75:5263. https://doi.org/10.1016/j.ijrefrig.2017.01.013

22. Tasnim SH, Mahmud S, Fraser RA (2012) Compressible pulsating convection through regular and random porous media: the thermoacoustic case. Heat Mass Transf 48:329-342. https://doi. org/10.1007/s00231-011-0884-7

23. Hariharan NM, Sivashanmugam P, Kasthurirengan S (2013) Experimental investigation of a thermoacoustic refrigerator driven by a standing wave twin thermoacoustic prime mover. Int J Refrig 36:2420-2425. https://doi.org/10.1016/j.ijref rig.2013.04.017

24. Alcock AC, Tartibu LK, Jen TC (2018) Experimental investigation of an adjustable thermoacoustically-driven thermoacoustic refrigerator. Int J Refrig 94:71-86. https://doi.org/10.1016/j.ijref rig.2018.07.015

25. Kajurek J, Rusowicz A, Grzebielec A (2017) The influence of stack position and acoustic frequency on the performance of thermoacoustic refrigerator with the standing wave. Arch Thermodyn 38:89-107. https://doi.org/10.1515/aoter-2017-0026

26. Tartibu LK (2016) Maximum cooling and maximum efficiency of thermoacoustic refrigerators. Heat Mass Transf 52:95-102. https ://doi.org/10.1007/s00231-015-1599-y

27. Tasnim SH, Mahmud S, Fraser RA (2011) Measurements of thermal field at stack extremities of a standing wave thermoacoustic heat pump. Front Heat Mass Transf 2:1-10. https://doi. org/10.5098/hmt.v2.1.3006

28. Setiawan I, Setia-Utomo AB (2013) The influence of the length and position of the stack on the performance of a thermoacoustic refrigerator. Disseration, Gadjah Mada University 
29. Tijani MEH, Zeegers JCH, De Waele ATAM (2002) Prandtl number and thermoacoustic refrigerators. J Acoust Soc Am 112:134-143. https://doi.org/10.1121/1.1489451

30. Wantha C, Assawamartbunlue K (2011) The impact of the resonance tube on performance of a thermoacoustic stack. Front Heat Mass Transf 2:1-8. https://doi.org/10.5098/hmt.v2.4.3006

31. Kajurek J, Rusowicz A (2018) Performance analysis of the thermoacoustic refrigerator with the standing wave and air as a working fluid. In: E3S web of conferences 44. https://doi. org/10.1051/e3sconf/20184400063

32. Nsofor EC, Ali A (2009) Experimental study on the performance of the thermoacoustic refrigerating system. Appl Therm Eng 29:2672-2679. https://doi.org/10.1016/j.appltherma leng.2008.12.036

33. Nayak BR, Pundarika G, Arya B (2017) Influence of stack geometry on the performance of thermoacoustic refrigerator. Sadhana Acad Proc Eng Sci 42:223-230. https://doi.org/10.1007/s1204 6-016-0585-5
34. Belcher JR, Slaton WV, Raspet R, Bass HE, Lightfoot J (1999) Working gases in thermoacoustic engines. J Acoust Soc Am 105:2677-2684. https://doi.org/10.1121/1.426884

35. Wheatley J, Hofler T, Swift GW, Migliori A (1985) Understanding some simple phenomena in thermoacoustics with applications to acoustical heat engines. Am J Phys 53:147-162. https://doi. org/10.1119/1.14100

36. Tijani MEH (2001) Loudspeaker-driven thermo-acousic refrigeration. Disseration, Technische Universiteit Eindhoven

37. Clark JP, Ward WC, Swif GW (2007) Design environment for low-amplitude thermoacoustic energy conversion (DeltaEC). J Acoust Soc Am 122:3014. https://doi.org/10.1121/1.2942768

Publisher's Note Springer Nature remains neutral with regard to jurisdictional claims in published maps and institutional affiliations. 